\title{
Production of inflectional morphology in a child with moderate hearing impairment
}

Thalia Hatzivasiliou, Marianna Hatzopoulou

Department of Logopaedics, Athens Metropolitan College, Greece

https://doi.org/10.36505/ExLing-2010/03/0015/000135

\begin{abstract}
The present study explores the production of bound morphemes in the speech of a nine-year old Greek child with moderate hearing impairment (HI). Data were obtained through the child's responses to subtests from the Diagnostic Verbal IQ Test (DVIQ, Stavrakaki et al. 2000) and samples of her speech. The analysis concerned the inflection of nouns, adjectives and verbs. The results showed that, even though the morphological features of names and verbs were marked correctly in the majority of utterances, errors were still observed in the child's speech. These errors concerned mainly the marking of the plural number in nouns and the perfective aspect in verbs.
\end{abstract}

Key words: hearing impairment, morphology, bound morphemes

\section{Introduction}

Studies on the acquisition of morphology in children with HI report that, even though grammatical morphemes are more easily perceived and processed by children with better residual hearing, there are still deficiencies observed in their speech.

Research on the production of morphemes by English deaf children using cochlear implants, which improve their residual hearing to moderate hearing loss, has shown that they exhibit difficulties in inflectional morphology and in particular that they have problems in marking the past tense of verbs and the plural number of nouns (Spencer et al. 1998; Svirsky et al. 2002). Similar findings have been reported for children with mild to moderate HI. Even though their language skills are comparable to those of their hearing peers, there are still some weaknesses observed in the production of morphemes (Elfenbein et al. 1994; Norbury et al. 2001; Hansson et al. 2007). Elfenbein et al. (1994) mention difficulties in marking verb tenses and plural number of irregular nouns, while Hansson et al. (2007) detected difficulties in marking tense of novel verbs.

\section{Aim of the study}

The aim of the present study was to assess the inflectional morphology of nouns, adjectives and verbs in a hard of hearing $(\mathrm{HH})$ child and to detect any possible error patterns. It was expected that morphological errors,

ExLing 2010: Proceedings of 3rd Tutorial and Research Workshop on Experimental Linguistics, 25-27 August, Athens, Greece 
which concern mainly verb tenses as well as plural forms of nouns, would be present in the child's speech.

\section{Method}

The participant of this study was a nine-year-old Greek girl (M.) with a rare congenital syndrome (Lacrimo-Auriculo-Dento-Digital syndrome - LADD), which has caused her conductive, moderate $\mathrm{HI}$ (hearing thresholds at $58,3 \mathrm{~dB}$ for the right and $68,3 \mathrm{~dB}$ for the left ear). M. has been using hearing aids since the age of seven. Data were collected by speech samples and by M.'s responses on subtests of the DVIQ (Stavrakaki et al. 2000). The analysis concerned the correct or erroneous use of inflectional morphemes in nouns, adjectives and verbs. The erroneous forms were further analyzed for markedness of the gender, number and case features in nouns and adjectives, and of the person, number, tense and aspect features in verbs.

\section{Results}

Measurements of the correct and erroneous tokens showed that there were a few errors presented in M.'s speech. M. produced a total of 32 erroneous forms, which correspond to $10.8 \%$ of her overall production of nouns, adjectives and verbs (295 uttered tokens). Even though M. produced more verbal (152) than nominal (143) tokens, she made more errors in nouns and adjectives than in verbs. The rate of errors in the production of nouns and adjectives reached 14\% (20 erroneous forms out of the 143 nominal tokens), while the rate of errors in the production of verbs was $7.9 \%$ (12 erroneous forms out of the 152 verbal tokens).

Table 1. Numbers and percentages of erroneous types of uttered names and verbs.

\begin{tabular}{|l|l|l|}
\hline \hline & No of errors/No of tokens & $\%$ of errors \\
\hline Nouns and adjectives & $20 / 143$ & $14.0 \%$ \\
\hline Verbs & $12 / 152$ & $7.9 \%$ \\
\hline Total & $32 / 295$ & $10.8 \%$ \\
\hline
\end{tabular}

The analysis of the errors regarding the production of nouns and adjectives revealed that the most frequent errors concerned the number feature, as 14 of the 20 erroneous nominal tokens were incorrect plural forms (9.8\% of the total production of nouns and adjectives). M. produced an incorrect plural suffix in 8 out of the 14 erroneous plurals, while she did not mark the plural feature in 5 instances. In one instance, she used a plural instead of the obligatory singular form. The remaining 6 incorrect forms concerned errors of the gender and case features. 
The most frequent error type in verbs concerned the aspect feature. M. made 5 errors of aspect out of the 12 erroneous verbal tokens (3.3\% of the total production of verbs). These errors referred to production of imperfective forms in contexts where the perfective form was expected. The rest of the errors were distributed across all other features: person 1, number $1, \operatorname{mood} 1$, tense 2 , voice 2 .

\section{Discussion}

The aforementioned results indicate that, even though morphological features were correctly marked in the majority of the child's utterances, there were still a few erroneous forms produced, as it was expected according to the literature (Elfenbein et al. 1994; Norbury et al. 2001; Hansson et al. 2007). Interestingly, in contrast to previous studies, the child, exposed to Greek, exhibited more difficulties regarding the morphology of nouns and adjectives than that of verbs. Studies in English have shown that children with HI have more difficulties in marking tense in verbs (Spencer et al. 1998; Svirsky et al. 2002). This discrepancy could possibly be attributed to the morphological characteristics of Greek compared to English.

A further qualitative analysis of the produced nominal errors concerning the incorrect marking of the plural form showed that they were probably overgeneralizations of specific morphological rules for the formation of plural number common in many nouns and adjectives. Namely, she overgeneralized the suffixes -/es/ and -/i/ for masculine and -/es/ for feminine names. M.'s main weakness in the morphology of verbs was detected in marking the perfective aspect of verbs. This pattern could also be considered as an overgeneralization strategy, as she marks only the tense morpheme to express both perfective and imperfective types, because she does not have the perfective morpheme at her disposal.

\section{Factors affecting the acquisition of morphology}

Various factors may have affected the acquisition of morphology in M.'s speech. The perceptual prominence hypothesis, which has been proposed for children with HI (Svirsky et al. 2002), may interpret some of M.'s difficulties, such as marking aspect, which concerns internal sound changes. However, it cannot account for all the errors observed in M.'s speech, such as plural marking in imparisyllabic nouns.

In the analysis, we speculated that grammatical and lexical familiarity affected the acquisition and production of morphemes. It was observed that the child produced more errors in categories that follow particular morphological rules, which do not occur frequently in Greek or in words that she did not recognize. Lexical familiarity has been suggested to affect the morphology of children with HI. Norbury et al. (2001) maintained that 
verbal morphology is affected by the verb's frequency, while Hansson et al. (2007) found that children with HI have difficulties in applying morphological rules to novel verbs.

\section{Conclusion}

Overall, the present study confirmed the previous findings on the acquisition of morphology of children with mild to moderate HI, showing that there are difficulties with the inflection of nouns, adjectives and verbs. The general assumption is that several linguistic factors, such as the perceptual salience, the grammatical and lexical familiarity of the linguistic input, may affect the morphological development of $\mathrm{HH}$ children. Therefore, it is important for children to be widely exposed to grammatical morphemes, especially those that are not frequently included in the linguistic input. Further research is needed on the grammatical development in Greek children with HI, as well as on the factors that affect the acquisition of morphology.

\section{References}

Elfenbein, J.L., Hardin-Jones, M.A., Davis, J.M. 1994. Oral communication skills of children who are hard of hearing. Journal of Speech and Hearing Research 37, 216-226.

Hansson, K., Sahlen, B., Maki-Torkko, E. 2007. Can a 'single hit' cause limitations in language development? A comparative study of Swedish children with hearing impairment and children with specific language impairment. International Journal of Language and Communication Disorders 42, 307-323.

Norbury, C.F., Bishop, D.V.M., Briscoe, J. 2001. Production of English finite verb morphology: A comparison of SLI and mild-moderate hearing impairment. Journal of Speech, Language and Hearing Research 44, 165-178.

Spencer, L.J., Tye-Murray, N., Tomblin, J.B. 1998. The production of English inflectional morphology, speech production and listening performance in children with cochlear implants. Ear and Hearing 19, 310-318.

Stavrakaki S., Tsimpli, I. M. 2000. Diagnostic Verbal IQ Test for Greek preschool and school age children: Standardization, statistical analysis, psychometric properties. In Proceedings of the 8th conference on speech therapy: 95-106. Athens: Ellinika Grammata.

Svirsky, M.A., Stallings, L.M, Lento, C.L., Ying, E., Leonard, L.B. 2002. Grammatical morphologic development in pediatric cochlear implant users may be affected by the perceptual prominence of the relevant markers. The Annals of Otology, Rhinology \& Laryngology 111, 109-112. 